\title{
A Pilot Study Comparing 2 Oxygen Delivery Methods for Patients' Comfort and Administration of Oxygen
}

\author{
Gil Jun Lee MSc, Sei Won Lee MD PhD, Yeon-Mok Oh MD PhD, Jae Seung Lee MD, \\ Sang-Do Lee MD PhD, Choongsoo S Shin PhD, and Tae Soo Lee PhD
}

\begin{abstract}
BACKGROUND: The traditional oxygen delivery methods for oxygen therapy are continuous flow oxygen (CFO) and demand oxygen delivery (DOD); however, oxygen waste is considerable in CFO, while DOD is uncomfortable for patients. Synchronized DOD (SDOD), which was designed to overcome the drawbacks of both CFO and DOD, supplies oxygen according to the patient's breathing pattern and the desired oxygen saving. This study was conducted to examine the overall performance of SDOD in terms of oxygen saturation $\left(\mathrm{S}_{\mathrm{pO}_{2}}\right)$, patients' comfort, and oxygen saving ratio (SR). METHODS: Study subjects were patients who required oxygen for COPD or pneumonia. Patients received oxygen through nasal prongs by CFO and SDOD for 30 min each. $S_{p_{2}}$ was measured every $10 \mathrm{~min}$ by pulse oximetry, and subjects recorded their level of comfort after $\mathbf{3 0}$ min. The flow of discharged oxygen was recorded to calculate SR. RESULTS: Ten subjects (median age, $68 \mathrm{y}$; range, 56-86 y) were enrolled. The $\mathrm{S}_{\mathrm{pO}_{2}}$ of patients during SDOD $(97 \pm 2 \%)$ was similar to that during CFO $(96 \pm 3 \%)$ with no statistically significant difference $(P=.53)$. Subjects reported SDOD to be more comfortable than CFO. The comfort score of subjects treated with SDOD was $7.05 \pm 2.07$ ( 0 : very uncomfortable, 10 : very comfortable); this was significantly higher $(P=.02)$ than the comfort score $(5.20 \pm 1.83)$ of subjects treated with CFO. The SR values set by clinicians were very similar to calculated SR values. CONCLUSIONS: SDOD appears to be more suitable for oxygen therapy than CFO when considering $\mathbf{S}_{\mathbf{p O}_{2}}$, patients' comfort, and SR. Key words: oxygen therapy; synchronized demand oxygen delivery; continuous flow oxygen; $S_{p o}$; discomfort; saving ratio. [Respir Care 2014;59(8):1191-1198. @ 2014 Daedalus Enterprises]
\end{abstract}

\section{Introduction}

Oxygen therapy is an essential treatment for patients with hypoxemia. Almost any kind of acute severe respiratory disease, such as pneumonia, COPD, exacerbation of

Mr GJ Lee is affiliated with the Graduate School of Sogang University; Drs SW Lee, Oh, JS Lee, and SD Lee are affiliated with the Department of Pulmonary and Critical Care Medicine, Asan Medical Center, University of Ulsan College of Medicine; and Drs Shin and TS Lee are affiliated with the Department of Mechanical Engineering, Sogang University, Seoul, Republic of Korea.

Mr GJ Lee and Dr SW Lee are co-first authors.

This study was supported by grant A091265-1002-0000100 from the Korea Healthcare Technology Research and Development Project, Ministry for Health, Welfare, and Family Affairs, Republic of Korea, and by research grant 201310020.01 from Sogang University. The authors have disclosed no conflicts of interest. asthma, and pulmonary edema, can cause hypoxemia and necessitate oxygen supplementation. Oxygen supplementation has also been proven to be effective in treatment of chronic respiratory diseases. Long-term oxygen therapy (LTOT) can improve the survival of COPD patients with severe hypoxemia. ${ }^{1-3}$ Ambulatory oxygen therapy also improves the exercise capacity of patients with interstitial lung diseases. ${ }^{4,5}$ Because patients who require such treatment need it most of the time in their daily lives, the comfort of oxygen treatment is very important to them.

There are 2 common methods for oxygen therapy: continuous flow oxygen (CFO) and demand oxygen delivery

\footnotetext{
Correspondence: Choongsoo S Shin PhD, Department of Mechanical Engineering, Sogang University, 1 Shinsu-Dong, Mapo-Gu, Seoul 121742, Republic of Korea. E-mail: cshin@ sogang.ac.kr.
}

DOI: $10.4187 /$ respcare. 02937 
(DOD); both methods have their inherent shortcomings. CFO involves considerable wastage of oxygen, because it supplies oxygen to patients during both inhalation and exhalation. CFO can restrict patients' activities, because a large oxygen cylinder is required to compensate for the wasteful continuous delivery of oxygen. DOD is a relatively recent oxygen-conserving strategy that reduces waste by delivering oxygen only during inhalation. As a result, DOD may also reduce the volume of oxygen needed for portable oxygen delivery with reduced number of oxygen cylinders. However, DOD can make patients uncomfortable because of the abrupt onset of oxygen supply at the beginning of inhalation.

Synchronized DOD (SDOD), a new oxygen delivery method, was developed and modified in our previous studies to overcome the drawbacks of both CFO and DOD.,67 SDOD offers patients a greater level of comfort by delivering oxygen in a smooth sinusoidal pattern that is synchronized to their breathing; in addition, the method permits oxygen conservation at different levels, which can be set by physicians or patients. ${ }^{6,7}$ According to a recent study by Lee et $a 1,{ }^{6}$ the majority of subjects who had no respiratory disease were more comfortable with the sinusoidal curve of SDOD than with the pulsed pattern of DOD. Nevertheless, study regarding the comfort level of patients with respiratory diseases is warranted because the comfort level of these patients might be different from that of subjects with no respiratory diseases.

The purpose of this study was to examine the overall performance of SDOD in terms of saturation of peripheral oxygen $\left(\mathrm{S}_{\mathrm{pO}_{2}}\right)$, patients' comfort level, and oxygen saving ratio (SR). The following 3 hypotheses were tested: (1) the $\mathrm{S}_{\mathrm{pO}_{2}}$ levels of patients are not different under CFO and SDOD, (2) the comfort levels of patients are not different under CFO and SDOD, and (3) SDOD could reduce oxygen waste.

\section{Methods}

\section{Subjects}

Patients who were admitted to the respiratory care center with $\mathrm{S}_{\mathrm{pO}_{2}}<90 \%$ on room air and who needed oxygen supplementation were enrolled for this study. This was a pilot study for which we planned to enroll only 10 subjects. The study was reviewed and approved by the institutional review board of Asan Medical Center (S20121904-0006). All subjects received a thorough explanation of the experimental procedures before signing informed consent forms.

\section{SDOD Device}

SDOD was designed, delivered, and studied using a bench model previously described in other studies ${ }^{6,7}$; thus,

\section{QUICK LOOK}

\section{Current knowledge}

Long-term oxygen therapy is commonly provided via nasal cannula as a low continuous flow or pulse dose oxygen delivery. Continuous flow oxygen is simple but wastes gas during the expiratory phase. Pulse dose oxygen delivery conserves gas, but can result in inadequate oxygen delivery as breathing frequency increases. Synchronized demand oxygen delivery is a method intended as an alternative to these 2 common methods.

\section{What this paper contributes to our knowledge}

In a small group of 10 subjects with hypoxemia, synchronized demand oxygen delivery resulted in similar oxygenation as measured by pulse oximetry when compared with continuous flow oxygen. Subjects reported improved comfort during synchronized demand oxygen delivery, and oxygen conservation was greater compared with continuous flow oxygen.

only a brief description is given here. SDOD, which uses a proportional valve to control oxygen flow, begins to supply oxygen just before inhalation in accordance with a sinusoidal discharge curve (Fig. 1), unlike DOD, which delivers oxygen abruptly only after sensing the onset of inhalation. SDOD monitors and records subjects' breathing signals by using the pressure sensor. Based on the recorded breathing signal, SDOD determines the time to open the valve to supply oxygen smoothly before the inhalation. Thus, SDOD is expected to improve patient comfort during oxygen therapy. Moreover, SDOD has been designed to deliver oxygen by 4 delivery modes to conserve oxygen according to set SR values. ${ }^{6,7}$

SDOD uses 4 oxygen delivery modes according to the set level of oxygen saving (Fig. 2). ${ }^{6,7}$ The first and second modes supply oxygen in a sigmoid pattern that peaks before inhalation. The third mode supplies oxygen before inhalation along a sigmoid curve that gives way to a pulsed pattern at the onset of inhalation. The fourth mode, which is similar to DOD, provides pulsed oxygen delivery at the beginning of inhalation. Therefore, SDOD can supply oxygen in different modes to achieve the desired SR set by patients or clinicians.

The SDOD device was reduced in size (length: $330 \mathrm{~mm}$, width: $230 \mathrm{~mm}$, height: $150 \mathrm{~mm}$, weight: $2.5 \mathrm{~kg}$ ) and made portable for this study when compared with the previous bench model of the SDOD, which consists of a mechanical respiratory simulator and the SDOD device. The device had previously been tested on the mechanical respiratory simulator, which mimics the human respiratory system, before being used on human subjects in the present study. 


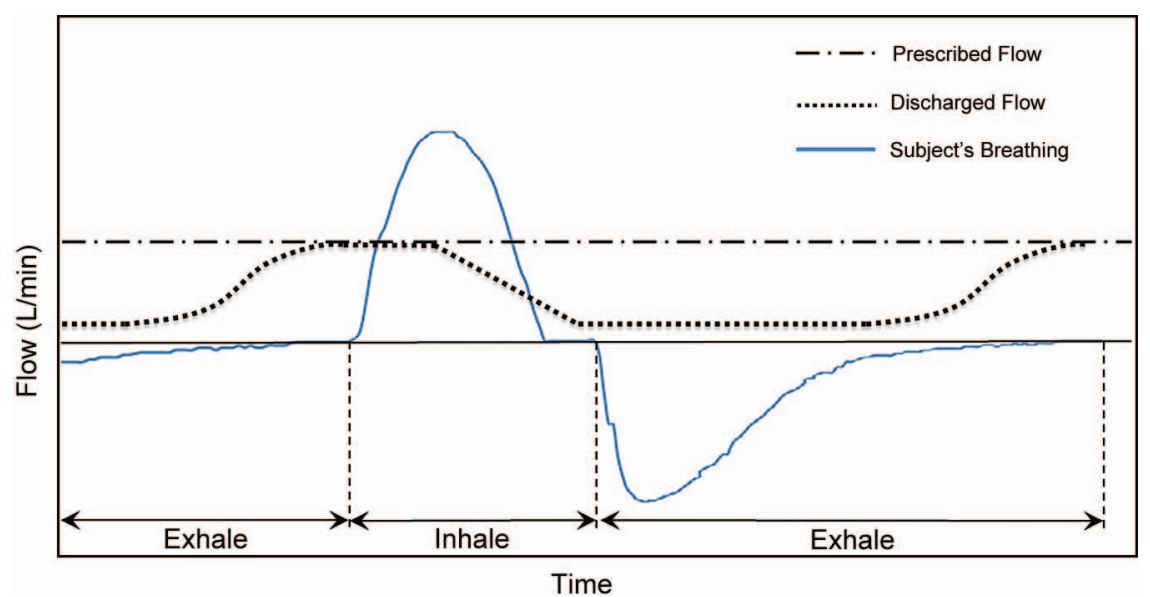

Fig. 1. Time-dependent oxygen discharge profile of synchronized demand oxygen delivery (SDOD). SDOD supplies oxygen in a sigmoidal pattern starting before inhalation, by synchronizing flow with the subject's breathing pattern.
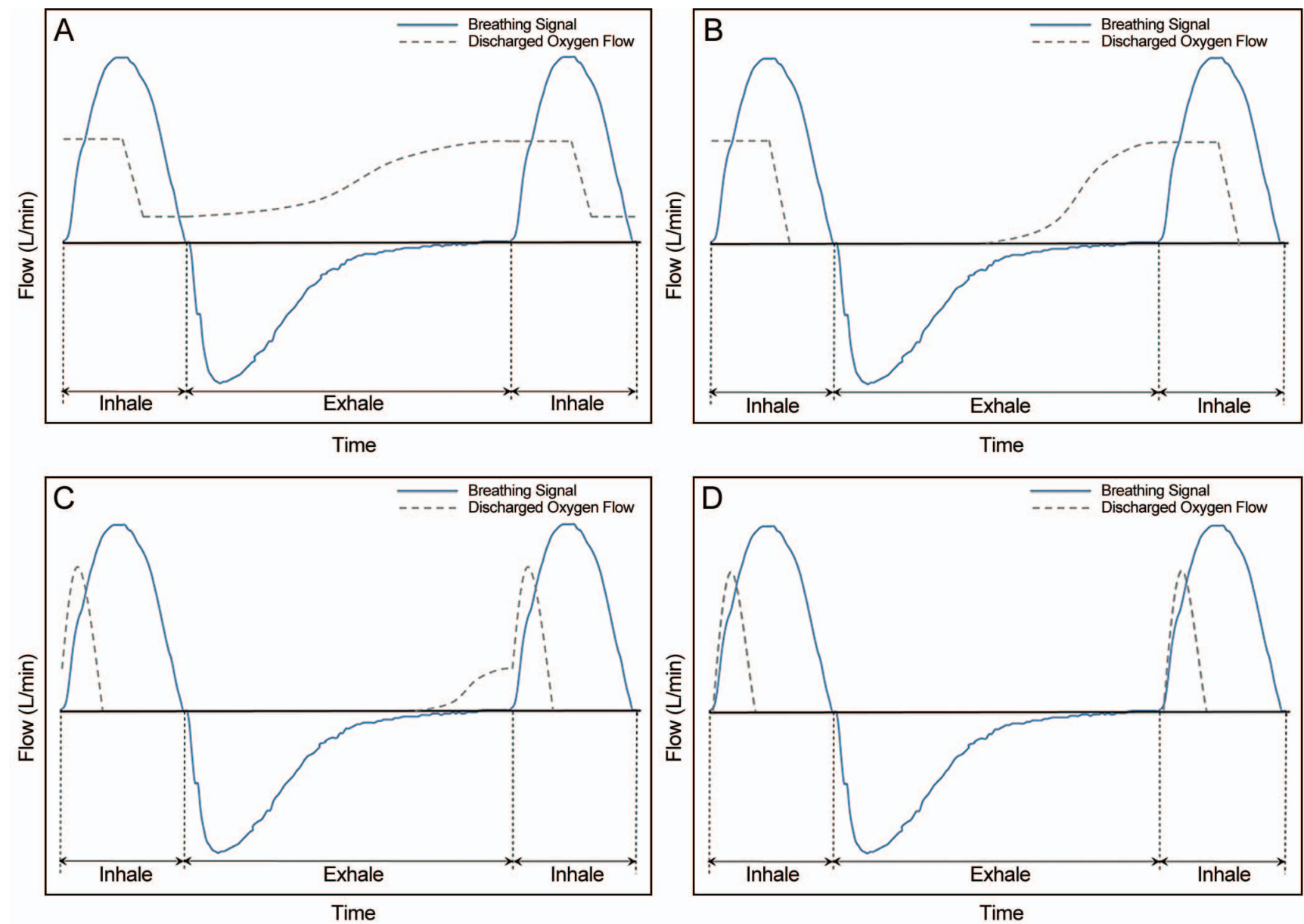

Fig. 2. Four oxygen delivery modes of synchronized demand oxygen delivery (SDOD). A and B: in the first and second modes, the oxygen discharge profile rises to the prescribed flow along a sigmoid curve before inhalation. $\mathrm{C}$ : in the third mode, oxygen supply pattern changes over from a sigmoid curve to a pulsed pattern at the onset of inhalation. D: in the fourth mode, pulsed oxygen delivery is used at the beginning of inhalation, the same way as in demand oxygen delivery. 


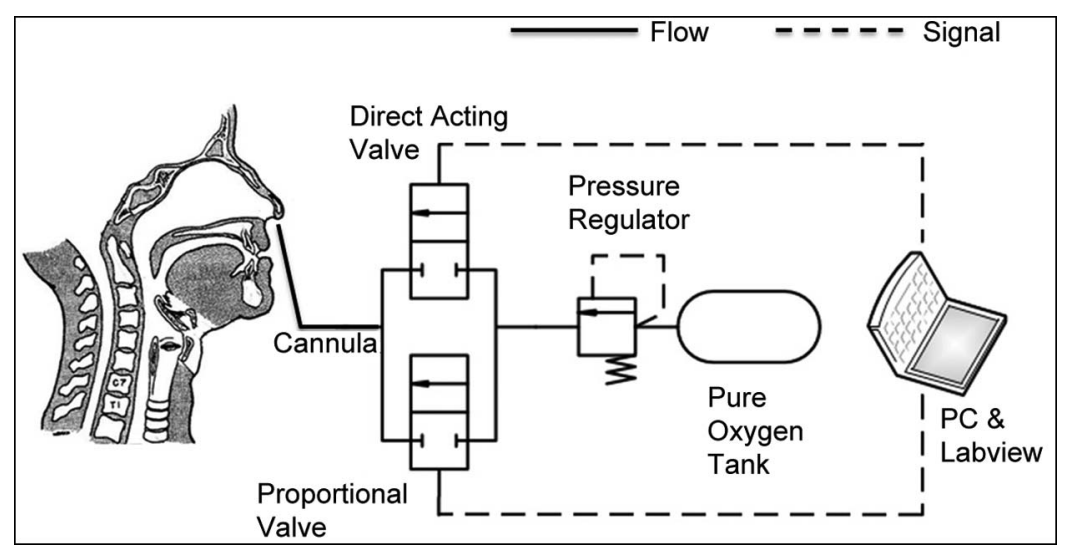

Fig. 3. Schematic of a synchronized demand oxygen delivery (SDOD) device used in the study. Subjects' breathing patterns were recorded and then predicted by the computer (PC) that implemented the SDOD algorithm. SDOD supplied oxygen in sigmoid and pulsed patterns by controlling 2 valves according to the subjects' breathing pattern and the set saving ratio.

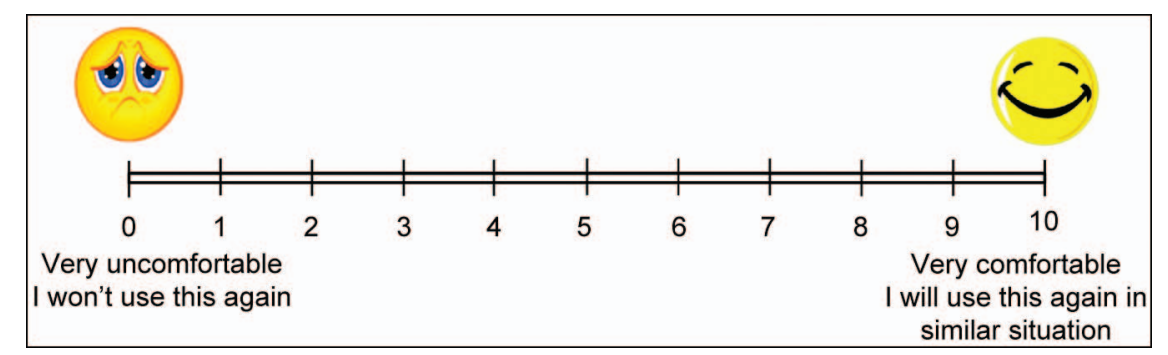

Fig. 4. Visual analog scale for subjects' comfort. Subjects scored their comfort level from 0 (very uncomfortable) to 10 (very comfortable) after 30 min of treatment with either continuous flow or synchronized demand oxygen delivery.

Figure 3 shows the schematic of the SDOD device, which consisted of 3 components: a direct-acting miniature solenoid valve (RB2-0139; Numatics, Sandusky, Michigan), which supplies oxygen in a pulsed pattern; a miniature proportional valve (VSonic-3S11-Q8; Parker, Cleveland, Ohio), which delivers oxygen in a sinusoidal pattern; and Labview 2010 system design software (National Instruments, Austin Texas), which controls the SDOD device.

SR is defined as the ratio of prescribed flow over supply flow. CFO does not save oxygen because it supplies oxygen continuously at the same flow as prescribed by the clinician. In other words, the SR of CFO is equal to 1 . On the other hand, an SR as high as 3.4 is seen in DOD, which was designed primarily to conserve oxygen. ${ }^{8} \mathrm{DOD}$ is therefore widely used for treating COPD patients, because the cost of oxygen is an important factor in the choice of treatment method.

The average supply rate was calculated by integrating the actual flow of oxygen over the period of each experiment; the prescribed flow was then divided by the calculated average supply rate to derive the actual SR. The actual SR was compared with the input SR, which is the SR inputted to the SDOD algorithm by patients, clinicians, or manufacturers.

\section{Study Design}

CFO and SDOD were tested on all 10 subjects in a randomized order. The study consisted of the following 3 steps: (1) Oxygen was administered to subjects via nasal prongs for $30 \mathrm{~min}$ by either CFO or SDOD; (2) $\mathrm{S}_{\mathrm{pO}_{2}}$ was measured every 10 min using a Fairy-A2 pulse oximeter (ShenZhen HeXin Zondan Medical Equipment, Guangdong, China); and (3) subjects were asked to score the degree of their discomfort on a scale from 0 (very uncomfortable) to 10 (very comfortable) after $30 \mathrm{~min}$ using the visual analog scale shown in Figure 4. The subjects were asked to remain seated or reclined with no physical activity during the oxygen treatment. Oxygen delivery was interrupted for 10 min between CFO and SDOD when subjects were switched over from one to the other, to allow $\mathrm{S}_{\mathrm{pO}_{2}}$ to drop to pretreatment levels. The SDOD device supplied oxygen by the first or second mode using sigmoid time-dependent oxygen profiles in this study. The actual flow of discharged oxygen was recorded to calculate the actual SR. ${ }^{7}$ Oxygen flow, when subjects were put on CFO, was set at the rate prescribed for individual subjects. 
Table 1. Subject Demographics

\begin{tabular}{|c|c|c|c|c|c|c|c|c|}
\hline $\begin{array}{l}\text { Subject } \\
\text { No. }\end{array}$ & Gender & $\begin{array}{c}\text { Age } \\
(\mathrm{y})\end{array}$ & $\begin{array}{l}\text { Main Causes } \\
\text { of Hypoxemia }\end{array}$ & $\begin{array}{c}\mathrm{FEV}_{1}, \mathrm{~L} \\
\text { (\% predicted) }\end{array}$ & $\begin{array}{c}\text { FVC, } \mathrm{L} \\
\text { (\% predicted) }\end{array}$ & $\begin{array}{l}\mathrm{FEV}_{1} / \\
\text { FVC }\end{array}$ & $\begin{array}{c}\text { LTOT Usage } \\
\text { Before Admission }\end{array}$ & $\begin{array}{l}\text { Prescription Flow } \\
\text { (L/min) }\end{array}$ \\
\hline 1 & Male & 66 & Pneumonia & $3.11(99)$ & $3.57(85)$ & 0.87 & No & 3 \\
\hline 2 & Female & 69 & Pneumonia & $2.08(97)$ & $2.60(88)$ & 0.80 & No & 3 \\
\hline 3 & Male & 70 & Pneumonia & ND & ND & ND & No & 1 \\
\hline 4 & Male & 75 & Pneumonia & $2.07(71)$ & $2.85(63)$ & 0.73 & No & 2 \\
\hline 5 & Male & 59 & Pneumonia & $0.95(30)$ & $0.97(24)$ & 0.98 & No & 3 \\
\hline 6 & Male & 85 & ILD & ND & ND & ND & No & 1 \\
\hline 7 & Male & 67 & Pneumonia & $0.91(28)$ & $1.77(40)$ & 0.51 & Yes & 1 \\
\hline 8 & Male & 66 & COPD & $0.83(37)$ & $1.90(60)$ & 0.44 & Yes & 2 \\
\hline 9 & Male & 56 & Pneumonia & ND & ND & ND & No & 2 \\
\hline 10 & Male & 86 & COPD & $0.87(32)$ & $2.02(45)$ & 0.43 & Yes & 1 \\
\hline $\begin{array}{l}\mathrm{FEV}_{1}=\text { for } \\
\mathrm{FVC}=\text { for } \\
\mathrm{LTOT}=\mathrm{l} \\
\mathrm{ND}=\text { no } \\
\mathrm{ILD}=\text { inte }\end{array}$ & $\begin{array}{l}\text { xpiratory vo } \\
\text { tal capacity } \\
\text { rm oxygen } \\
\text { railable } \\
1 \text { lung disea }\end{array}$ & $\begin{array}{l}\text { in } 1 \mathrm{~s} \\
\mathrm{y}\end{array}$ & & & & & & \\
\hline
\end{tabular}

\section{Statistical Analysis}

$\mathrm{S}_{\mathrm{pO}_{2}}$ and the comfort level of subjects were compared for the 2 treatment methods by Wilcoxon signed rank test. All statistical analyses were performed using SPSS 18.0 (SPSS, Chicago, Illinois). A $P$ value of $<.05$ was considered statistically significant.

\section{Results}

Ten subjects were enrolled in the study. There were 9 men and 1 woman, with a median age of $68 \mathrm{y}$, ranging from 56 to $86 \mathrm{y}$. All subjects were examined after clinical stabilization with regular breathing and $\mathrm{S}_{\mathrm{pO}_{2}} \geq 90 \%$ by low oxygen delivery via nasal prongs in bed. The demographic data for all subjects are summarized in Table 1.

The $\mathrm{S}_{\mathrm{pO}_{2}}$ of each subject after $30 \mathrm{~min}$ of treatment by each oxygen delivery method is summarized in Figure 5. The mean $\mathrm{S}_{\mathrm{pO}_{2}}$, as measured by pulse oximetry after $30 \mathrm{~min}$ of oxygen treatment by SDOD, was $97 \pm 2 \%$. When compared with the value of $\mathrm{S}_{\mathrm{pO}_{2}}$ under $\mathrm{CFO}$, there was no difference in $\mathrm{S}_{\mathrm{pO}_{2}}$ between the 2 methods $(P=.53)$.

Subjects found SDOD to be more comfortable than CFO. The comfort score of subjects after $30 \mathrm{~min}$ of oxygen treatment by SDOD was $7.05 \pm 2.07$; this was significantly higher $(P=.02)$ than the comfort score of $5.20 \pm 1.83$ after CFO treatment (Fig. 5).

The actual SR values achieved by SDOD, as calculated from the recorded flow of oxygen, were very similar to the SR values inputted by clinicians (Fig. 6). This indicates that SDOD can be adjusted to achieve set levels of oxygen saving preferred by users. The mean actual SR was 1.90; this means that a supply flow of approximately $1 \mathrm{~L} / \mathrm{min}$, delivered by the SDOD method, should be enough to treat a patient who has been prescribed oxygen at the rate of $1.9 \mathrm{~L} / \mathrm{min}$.

\section{Discussion}

The present study investigated the performance of the SDOD device, which was driven by a computer running Labview (National Instruments, Austin, Texas), in subjects with respiratory disease by conducting a bedside bench study. The $\mathrm{S}_{\mathrm{pO}_{2}}$ of subjects on SDOD was compared with that of the same subjects on CFO, to assess the efficacy of SDOD in subjects with COPD or pneumonia. Our results show that 30 min of oxygen treatment by SDOD could raise $\mathrm{S}_{\mathrm{pO}_{2}}$ to a desirable level. Furthermore, mean $\mathrm{S}_{\mathrm{pO}_{2}}$ in subjects on SDOD was statistically similar to that in subjects on CFO. These results indicate that the SDOD device does indeed provide patients with an adequate supply of oxygen, and thus could replace $\mathrm{CFO}$ as the preferred method of oxygen therapy.

Almost all subjects found SDOD to be more comfortable than CFO after 30 min of treatment, and the difference was statistically significant (Fig. 4). The level of comfort can be an important determinant in choosing the method of oxygen delivery, and SDOD appears to be more suitable than CFO based on the results of this study, especially for patients with COPD, most of whom require long-term treatment.

The cost of oxygen has become a significant issue in view of the widespread application of LTOT, and SDOD assumes importance in this scenario by providing the additional benefit of oxygen conservation. The average SR of SDOD was approximately 2 in our study. The SR of SDOD is lower than that of DOD devices that focus entirely on conserving oxygen. However, SDOD offers the 

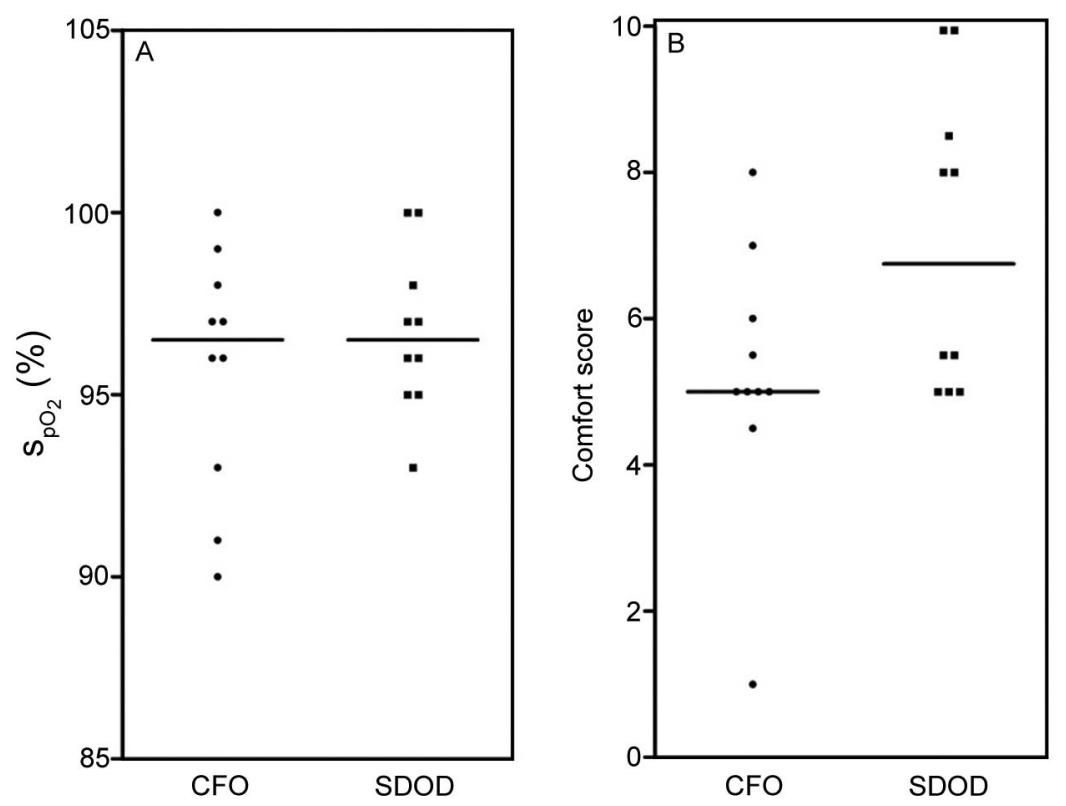

Fig. 5. Oxygen saturation $\left(\mathrm{S}_{\mathrm{pO}_{2}}\right)$ and comfort levels of subjects on synchronized demand oxygen delivery (SDOD) and continuous flow oxygen (CFO). A: oxygen saturation levels for both methods $(P=.16)$. B: comfort levels for both methods. Study participants scored their comfort level on a scale of 0 to $10(0=$ very uncomfortable; $10=$ very comfortable $)$ after 30 min of treatment with either CFO or SDOD $(P=$ .02).

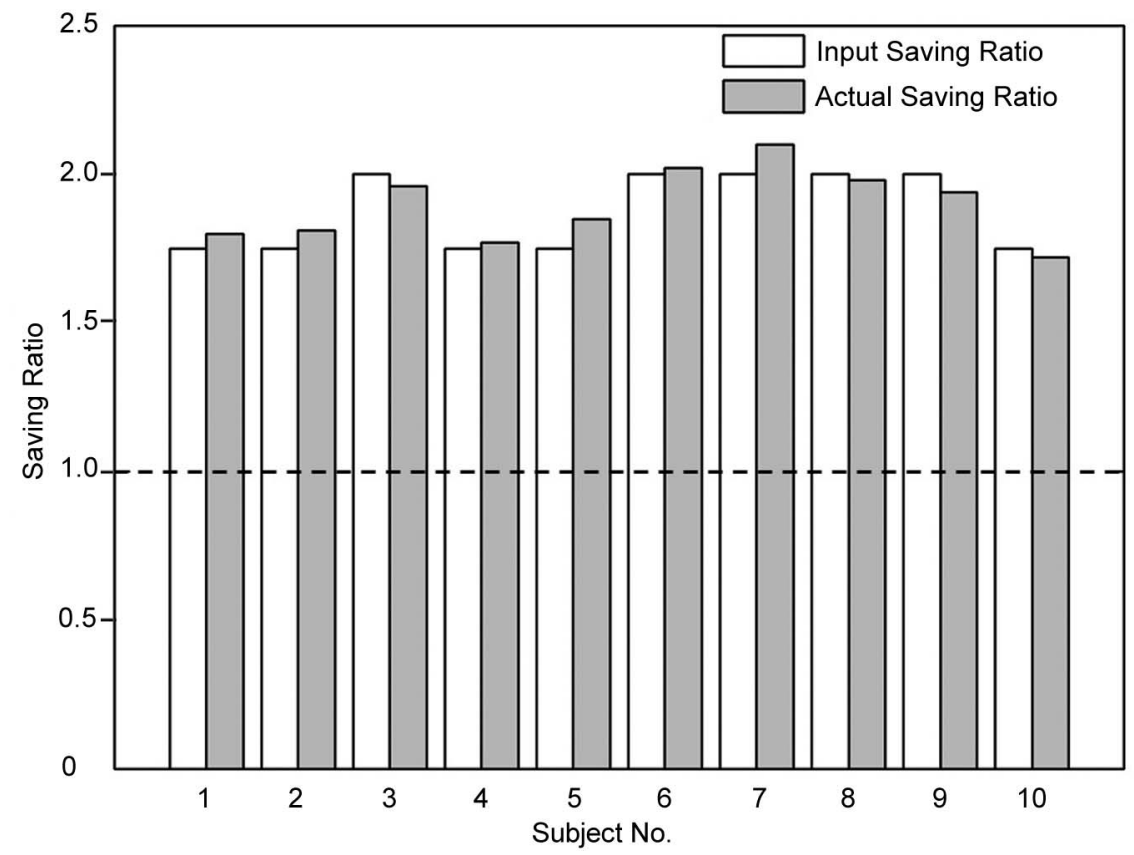

Fig. 6. Set saving ratio and actual saving ratio during $30 \mathrm{~min}$ of oxygen treatment with synchronized demand oxygen delivery (SDOD). The saving ratio is defined as the ratio of the prescribed flow over the rate of actual supply rate integrated over the breathing cycle (saving ratio = prescribed flow/supply flow); the higher the saving ratio, the more the oxygen conserved. The saving ratio of continuous flow oxygen (CFO) is equal to 1 (dotted line) because CFO supplies oxygen continuously at the prescribed rate.

simultaneous advantages of oxygen conservation and a high level of comfort during treatment.

SDOD allows smaller oxygen carriers to be used because it consumes less oxygen than CFO. The resultant decrease in the size of the oxygen cylinder can greatly improve patients' mobility. Thus, SDOD not only makes oxygen treatment more cost-effective as a result of less wasted oxygen, it also enhances the ability of patients to 


\section{Oxygen Delivery and Patient Comfort}

participate in physical activities because the size of the oxygen cylinder could be reduced using SDOD.

Oxygen was supplied to the subject using the first or second mode of SDOD to remove the effect of DOD's pulsed patterns. The third and fourth modes of SDOD are very similar to the current DOD devices, because these modes supply oxygen using pulsed patterns at the onset of inhalation. Because the purpose of this study was to compare the $\mathrm{S}_{\mathrm{pO}_{2}}$ level and comfort of the subjects under treatment with SDOD and CFO, we tested only the first or second mode of SDOD, which supplied oxygen without the pulsed pattern. In addition, the difference between the first and second mode of SDOD is whether oxygen is supplied during exhalation or not. As shown in Figure 2, in the first mode, oxygen is supplied with a base oxygen flow lower than the prescribed flow, and then oxygen flow smoothly increases to the prescribed flow before inhalation. In the second mode, oxygen flow rises from zero to the prescribed flow according to the smooth oxygen discharge curve. Therefore, the SR of the second mode is higher than that of the first mode. Because the first and second mode of SDOD supply oxygen according to a very similar oxygen discharge pattern, it seems that the patients' feeling of comfort under the 2 modes does not vary.

The size of the SDOD device can be reduced to that of the current DOD device attached to the oxygen cylinder. The SDOD device used in this study is still a prototype driven and controlled by a laptop computer with Labview software. Because the SDOD algorithm can be programmed into a microprocessor, the SDOD device can be driven by the programmed microprocessor without the laptop. The SDOD prototype was powered by alternating current; however, the power source is able to be replaced with a battery as in current DOD devices. Thus, the expected size of the SDOD device targeted to a marketplace will be more compact and much lighter than that of the current prototype.

The SDOD device might be used for LTOT by simply plugging it to the oxygen cylinder. As a result, oxygen costs can be saved in hospitals by reduced oxygen waste by SDOD. Moreover, oxygen can be supplied to patients more comfortably at home or other places, if the SDOD is portable and commercialized in the future.

One limitation of the current study is the small number of subjects (10 subjects) with various clinical conditions involved, only some of whom were receiving LTOT before admission; further studies with more subjects with specific conditions and diseases are therefore needed to prove the effectiveness of SDOD more objectively. However, despite the small number of subjects in our study, SDOD showed statistically significant advantages over $\mathrm{CFO}$ in terms of subject comfort, while maintaining $\mathrm{S}_{\mathrm{pO}_{2}}$ at similar levels.

Another limitation of this study is that $\mathrm{S}_{\mathrm{pO}_{2}}$ was measured only by pulse oximetry without measuring arterial oxygen saturation $\left(\mathrm{S}_{\mathrm{aO}_{2}}\right)$ or arterial oxygen pressure. There is controversy over the adequacy of pulse oximetry for evaluating patients under LTOT.9-11 Yelderman and New ${ }^{12}$ and Mendelson et $\mathrm{al}^{13}$ have shown that pulse oximetry measures oxygen saturation accurately in healthy subjects. Pulse oximetry has also been shown to measure oxygen saturation accurately in critically ill patients. ${ }^{14}$

Our study is also limited by the relatively short-term use of SDOD for just $30 \mathrm{~min}$, since the comfort level of patients treated with SDOD for hours or days may be quite different. It is thus necessary to conduct studies of longer duration to compare SDOD and CFO. In addition, the subjects were asked to sit or recline during the test. Under the activities of daily living, the breathing patterns of the subjects can change when compared with resting breathing patterns. Because of this, another experiment incorporating the subjects' physical activities is warranted to examine the performance of SDOD more thoroughly. Furthermore, during sleep, demand valves do not work occasionally, due to the breathing pattern and triggering sensitivity of the oxygen-conserving devices. ${ }^{15}$ Thus, adequacy and safety of the proportional valve used in this study should be carefully assessed.

\section{Conclusions}

We conducted the present study to document the effectiveness of oxygen therapy by SDOD in comparison with CFO. Our results show that the difference in $\mathrm{S}_{\mathrm{pO}_{2}}$ between CFO and SDOD was not statistically significant; in other words, SDOD could elevate $\mathrm{S}_{\mathrm{pO}_{2}}$ to desirable levels just as well as CFO did. However, subjects found SDOD to be more comfortable than CFO. Thus, SDOD has the potential to replace $\mathrm{CFO}$, especially in the care of patients who need LTOT, by offering a greater level of comfort with the additional benefit of conserving oxygen, while providing equivalent $\mathrm{S}_{\mathrm{pO}_{2}}$ levels. However, further studies need to be conducted on a larger number of subjects treated with long-term SDOD, to validate the results of the present study, which was done with just 10 subjects given oxygen treatment for only $30 \mathrm{~min}$.

\section{REFERENCES}

1. Nocturnal Oxygen Therapy Trial Group. Continuous or nocturnal oxygen therapy in hypoxemic chronic obstructive lung disease: a clinical trial. Ann Intern Med 1980;93(3):391-398.

2. Long term domiciliary oxygen therapy in chronic hypoxic cor pulmonale complicating chronic bronchitis and emphysema: report of the Medical Research Council Working Party. Lancet 1981;317:681686.

3. Bowton DL, Scuderi PE, Haponik EF. The incidence and effect on outcome of hypoxemia in hospitalized medical patients. Am J Med 1994;97(1):38-46. 


\section{Oxygen Delivery and Patient Comfort}

4. Visca D, Montgomery A, de Lauretis A, Sestini P, Soteriou H, Maher TM, et al. Ambulatory oxygen in interstitial lung disease. Eur Respir J 2011;38(4):987-990.

5. Eaton T, Young P, Milne D, Wells AU. Six-minute walk, maximal exercise tests: reproducibility in fibrotic interstitial pneumonia. Am J Respir Crit Care Med 2005;171(10):1150-1157.

6. Lee GJ, Oh YM, Oh SK, Shin CS, Lee TS. Synchronization of oxygen delivery with breathing pattern for enhanced comfort: a bench study. Respir Care 2013;58(3):498-506.

7. Lee GJ, Cha SK, Oh SK, Shin CS, Lee TS. Synchronized oxygen delivery and its optimization method: a bench study. Int J Precision Engineering Manufacturing 2013;14(4):663-670.

8. Castillo D, Güell R, Casan P. Oxygen-conserving systems: a forgotten resource. Arch Bronconeumol 2007;43(1):40-45.

9. Carlin BW, Clausen JL, Ries AL. The use of cutaneous oximetry in the prescription of long-term oxygen therapy. Chest 1988;94(2):239241
10. Pierson DJ. Pulse oximetry versus arterial blood gas specimens in long-term oxygen therapy. Lung 1990;168 Suppl:782-788.

11. Roberts CM, Bugler JR, Melchor R, Hetzel MR, Spiro SG. Value of pulse oximetry in screening for long-term oxygen therapy requirement. Eur Respir J 1993;6(4):559-562.

12. Yelderman M, New W Jr. Evaluation of pulse oximetry. Anesthesiology 1983;59(4):349-352.

13. Mendelson Y, Kent JC, Shahnarian AA, Welch GW, Giasi RM Evaluation of the Datascope ACCUSAT pulse oximeter in healthy adults. J Clin Monit 1988;4(1):59-63.

14. Mihm FG, Halperin BD. Noninvasive detection of profound arterial desaturations using a pulse oximetry device. Anesthesiology 1985; 62(1):85-87.

15. Cuvelier A, Muir JF, Czernichow P, Vavasseur E, Portier F, Benhamou D, Samson-Dolfuss D. Nocturnal efficiency and tolerance of a demand oxygen delivery system in COPD patients with nocturnal hypoxemia. Chest 1999;116(1):22-29.

This article is approved for Continuing Respiratory Care Education credit. For information and to obtain your CRCE

(free to AARC members) visit 